\title{
Aproximación a los intereses e inquietudes de los profesores en formación inicial de español a partir del análisis de los portafolios reflexivos en lo que se refiere a «Dar clase»
}

\section{Mónica Barros Lorenzo}

Faculdade de Letras da Universidade do Porto, Portugal mlorenzo@letras.up.pt

\section{Pilar Nicolás Martínez}

Faculdade de Letras da Universidade do Porto y Centro de Investigação Transdisciplinar «Cultura, Espaço e Memória» (CITCEM), Portugal mmartinez@letras.up.pt

Marta Pazos Anido

Faculdade de Letras da Universidade do Porto, Portugal manido@letras.up.pt

\section{Resumen}

La investigación que se presenta a continuación se enmarca en los másteres profesionalizantes en enseñanza de lenguas (portugués como lengua materna + lengua extranjera o dos lenguas extranjeras), en la que la lengua extranjera (o una de ellas) es el español. Esta formación, impartida en la Faculdade de Letras da Universidade do Porto, habilita en Portugal para ejercer la profesión docente en el tercer ciclo de la enseñanza básica y en secundaria.

Durante el segundo año de estos ciclos de estudios universitarios se contempla la realización de prácticas docentes en institutos; además, el reglamento de estos másteres solicita a los profesores en prácticas la elaboración de un portafolio asociado a esta experiencia. En el área de español, este documento se concibe como un portafolio de naturaleza reflexiva que, para una de sus secciones, toma como base el documento Portafolio europeo para futuros profesores de idiomas (PEFPI).

Para la realización de este estudio, se ha recogido un total de 60 portafolios que comprenden el periodo de 2014 a 2017. De entre ellos, se han seleccionado los descriptores relativos a una de las siete categorías del PEFPI: «Dar clase»; con el fin de analizar los aspectos didácticos en los cuales los futuros docentes de español centran sus preocupaciones e intereses durante su periodo formativo. 
Palabras clave: «Dar clase», Portfolio Europeo para futuros profesores de idiomas, portafolio reflexivo, preocupaciones de los futuros docentes, profesores en formación inicial de español

\begin{abstract}
The research presented below is part of the professional Masters in language teaching (Portuguese as a mother tongue + foreign language or two foreign languages), in which the foreign language (or one of them) is Spanish, at the Faculty of Arts and Humanities of the University of Porto. This training enables future teachers to practice the teaching profession in the third cycle of basic education and secondary education in Portugal.

During the second year of these postgraduate courses, teaching practice in secondary schools is envisaged; in addition, the regulation of these Master's degrees requires the trainees to prepare a portfolio related to this experience. In the area of Spanish, this document is conceived as a portfolio of reflective nature and one of its sections is based on the European Portfolio for Student Teachers of Languages (EPOSTL).

To carry out this study, a total of 60 portfolios have been collected, covering the period from 2014 to 2017. Among them, descriptors have been selected for one of the seven EPOSTL categories: "Conducting a lesson"; in order to analyse the didactic aspects in which future Spanish teachers focus their concerns and interests on during their training period.
\end{abstract}

Keywords: "Conducting a lesson", European Portfolio for Student Teachers of Languages, reflective portfolio, concerns of future teachers, teachers in initial Spanish training

\title{
1 - Introducción
}

Desde inicios del siglo XX las inquietudes o preocupaciones de los profesores han sido uno de los temas frecuentes de investigación en el área de la educación, según explica Simon Veenman (1984), aunque es a partir de los años 60 cuando se comienzan a recoger datos más concretos sobre esta cuestión. Un ejemplo de ello es el reconocido modelo de desarrollo docente basado en las preocupaciones de los profesores principiantes de Frances F. Fuller desarrollado a mediados de la década de 1950. Fuller 
BARROS LORENZO, Mónica; Nicolás Martínez, Pilar; Pazos Anido, Marta - Aproximación a los intereses e inquietudes de los profesores... Para lá da tarefa: implicar os estudantes na aprendizagem de línguas estrangeiras no ensino superior. Porto: FLUP, 2019, pp. 44-67 DOI: https://doi.org/10.21747/9789898969217/paraa3

(1969) distinguía tres etapas: una primera fase en la que priman las preocupaciones de los docentes sobre sí mismos, una segunda etapa en la que prevalece la preocupación sobre las tareas y, una tercera etapa, centrada en los alumnos. A partir de este trabajo surgen otras propuestas, como la de Fuller y Brown (1975) y la de Gene Hall y Shirley Hord (1987) que desarrollan durante décadas el programa denominado The ConcernsBased Adoption Model, conocido también por sus siglas $C B A M^{1}$, cuyo marco conceptual tiene muy presente que cada persona, en este caso cada docente, responde a un nuevo contexto con actitudes y creencias únicas, destacando la llamada «Stages of Concern» o etapa de preocupación. En esta línea argumental, Hall y Hord definen una preocupación como «la representación compuesta de sentimientos, inquietudes, pensamientos y consideraciones dadas a una cuestión particular o a una tarea» (Hall \& Hord, 1987, citado por Marcelo, Mayor \& Sánchez, 1995, p. 154) y, según los mismos autores, este concepto de «preocupación» adquiere un papel destacado «en la medida en que asume que hay que atender a las necesidades y demandas específicas de los profesores que se implican en procesos de cambio.» (Marcelo, Mayor \& Sánchez, 1995, p. 154).

Por otro lado, Veenman (en su estudio ya mencionado de 1984) categoriza los problemas identificados por los profesores principiantes durante los primeros años de enseñanza, a partir del análisis de 83 investigaciones (1984, p. 152) realizados en diferentes países. El resultado de este estudio se traslada a una tabla en la que se recoge una lista de los veinticuatro problemas que registran una mayor frecuencia.

\begin{tabular}{|c|c|c|c|c|c|c|c|c|c|c|c|c|c|}
\hline \multirow{2}{*}{$\begin{array}{l}\text { Rank or- } \\
\text { der }^{a}\end{array}$} & \multirow{2}{*}{ Problems } & \multicolumn{3}{|c|}{ All studies $(N=91)$} & \multicolumn{3}{|c|}{$\begin{array}{l}\text { Elementary level } \\
\qquad(N=28)\end{array}$} & \multicolumn{3}{|c|}{ Secondary level $(N=27)$} & \multicolumn{3}{|c|}{$\begin{array}{l}\text { Elem. \& secondary } \\
\quad(N=36)\end{array}$} \\
\hline & & Freq. & Med. & Q & Freq. & Med. & $\mathrm{Q}$ & Freq. & Med. & Q & Freq. & Med. & Q \\
\hline 1 & Classroom discipline & 77 & 13.0 & 1.4 & 22 & 12.3 & 1.4 & 23 & 13.5 & 1.3 & 32 & 12.5 & 1.5 \\
\hline 2 & Motivating students & 48 & 12.8 & 1.9 & 11 & 12.0 & 2.5 & 16 & 14.0 & 1.5 & 21 & 12.0 & 1.8 \\
\hline 3 & Dealing with individual differences & 43 & 13.0 & 1.5 & 15 & 12.0 & 1.3 & 12 & 13.8 & 0.5 & 16 & 12.0 & 1.5 \\
\hline 4.5 & Assessing students' work & 31 & 10.0 & 2.0 & 9 & 9.0 & 2.5 & 8 & 9.0 & 2.7 & 14 & 10.5 & 2.3 \\
\hline 4.5 & Relations with parents & 31 & 9.0 & 3.5 & 11 & 11.5 & 2.5 & 4 & 6.0 & 1.8 & 16 & 9.3 & 3.9 \\
\hline 6.5 & Organization of class work & 27 & 12.5 & 2.8 & 10 & 12.8 & 3.4 & 2 & 13.8 & 0.3 & 15 & 11.0 & 2.3 \\
\hline 6.5 & Insufficient materials and supplies & 27 & 11.0 & 2.5 & 9 & 13.0 & 1.8 & 6 & 10.5 & 2.5 & 12 & 9.3 & 3.3 \\
\hline 8 & $\begin{array}{l}\text { Dealing with problems of individual } \\
\text { students }\end{array}$ & 26 & 12.5 & 1.5 & 7 & 11.0 & 1.3 & 8 & 13.0 & 1.6 & 11 & 13.0 & 1.0 \\
\hline 9 & $\begin{array}{l}\text { Heavy teaching load resulting in in- } \\
\text { sufficient prep. time }\end{array}$ & 25 & 12.0 & 2.3 & 6 & 12.5 & 1.8 & 7 & 10.0 & 2.5 & 12 & 12.0 & 2.1 \\
\hline 10 & Relations with colleagues & 24 & 8.0 & 3.0 & 6 & 9.3 & 3.4 & 8 & 10.5 & 3.4 & 10 & 6.0 & 2.3 \\
\hline 11 & Planning of lessons and schooldays & 22 & 11.8 & 2.6 & 6 & 11.3 & 3.4 & 4 & 12.5 & 1.6 & 12 & 11.8 & 3.6 \\
\hline 12 & $\begin{array}{l}\text { Effective use of different teaching } \\
\text { methods }\end{array}$ & 20 & 12.0 & 3.6 & 5 & 12.5 & 5.1 & 6 & 11.5 & 2.3 & 9 & 12.0 & 2.6 \\
\hline
\end{tabular}

1 Se puede consultar este modelo en http://www.sedl.org/cbam/ y su actualización en https://www.air.org/resource/concerns-based-adoption-modelcbam 


\begin{tabular}{|c|c|c|c|c|c|c|c|c|c|c|c|c|c|}
\hline 13 & $\begin{array}{l}\text { Awareness of school policies and } \\
\text { rules }\end{array}$ & 19 & 11.0 & 3.0 & 6 & 10.5 & 4.7 & 5 & 13.5 & 3.5 & 8 & 11.0 & 1.9 \\
\hline 14 & $\begin{array}{l}\text { Determining learning level of stu- } \\
\text { dents }\end{array}$ & 16 & 10.5 & 2.8 & 3 & 13.0 & 2.8 & 6 & 9.0 & 2.7 & 7 & 11.0 & 3.3 \\
\hline 16 & Knowledge of subject matter & 15 & 11.0 & 1.5 & 5 & 11.0 & 2.1 & 5 & 11.0 & 1.6 & 5 & 8.5 & 2.0 \\
\hline 16 & Burden of clerical work & 15 & 9.0 & 1.8 & 4 & 11.0 & 2.3 & 1 & 7.0 & - & 10 & 9.0 & 1.4 \\
\hline 16 & $\begin{array}{l}\text { Relations with principals/adminis- } \\
\text { trators }\end{array}$ & 15 & 9.0 & 3.0 & 4 & 8.5 & 3.3 & 4 & 8.5 & 3.1 & 7 & 9.0 & 3.5 \\
\hline 18 & Inadequate school equipment & 14 & 11.0 & 2.6 & 6 & 10.5 & 3.5 & 2 & 11.3 & 0.8 & 6 & 11.0 & 3.1 \\
\hline 19 & Dealing with slow learners & 13 & 12.0 & 1.4 & 3 & 11.0 & 4.0 & 6 & 12.0 & 1.7 & 4 & 13.0 & 1.8 \\
\hline 20 & $\begin{array}{l}\text { Dealing with students of different } \\
\text { cultures and deprived back- } \\
\text { grounds }\end{array}$ & 12 & 9.0 & 2.6 & 3 & 3.0 & 4.0 & 2 & 9.0 & 0.0 & 7 & 9.0 & 2.5 \\
\hline 21 & $\begin{array}{l}\text { Effective use of textbooks and cur- } \\
\text { riculum guides }\end{array}$ & 11 & 8.5 & 3.5 & 3 & 6.5 & 5.5 & 2 & 14.0 & 1.0 & 6 & 7.8 & 2.4 \\
\hline 22 & Lack of spare time & 10 & 11.0 & 2.3 & 1 & 5.0 & - & 2 & 11.0 & 1.0 & 7 & 11.0 & 2.3 \\
\hline 23 & Inadequate guidance $\&$ support & 9 & 8.0 & 2.5 & 2 & 9.8 & 1.8 & 1 & 14.0 & - & 6 & 8.0 & 2.2 \\
\hline 24 & Large class size & 8 & 9.5 & 2.4 & 3 & 9.0 & 3.5 & 0 & - & - & 5 & 10.0 & 2.1 \\
\hline
\end{tabular}

Figura 1. Los 24 problemas más frecuentes según los profesores principiantes (Veenman,1984, pp. 154-155).

Centrándonos en la observación de algunas de sus conclusiones, llama la atención que Veenman destaque que los datos obtenidos no difieren significativamente entre los profesores con experiencia docente y los que no la tienen, así como entre los profesores de primaria y secundaria; pese a que señala que el orden de los elementos o su prioridad pueden variar, consideración relacionada con las conclusiones de Fuller en las que identificaba las tres etapas ya mencionadas por las que pasaban los docentes principiantes. De hecho, se podría hablar de unas preocupaciones «esenciales» para los docentes, sea cual sea su contexto educativo o experiencia, pues (según la literatura revisada) desde la década de los 30 del pasado siglo, pasando por los años 60, 70 y 80 , y llegando a las investigaciones más recientes sobre este tema se constata que no hay cambios sustanciales y que el listado de Veenman sigue siendo vigente y se cita con regularidad en la actualidad (Eirín, García Ruso \& Montero, 2009; Flores, 1999; Reyes, 2011).

En esta línea de investigación se encuadra este trabajo, pues en nuestro caso concreto (como formadoras de futuros profesores en los másteres de la Faculdade de Letras da Universidade do Porto -a partir de ahora FLUP-) nos interesa saber reconocer y reflexionar sobre las inquietudes que surgen durante los primeros años de profesionalización en la enseñanza, con el fin de detectar posibles aspectos de mejora en los programas de formación inicial de profesores en los que estamos integradas, para así allanar la transición de nuestros estudiantes a la realidad del aula (Flores, 1999, p. 176; Veenman, 1984, p. 143). 


\section{2 - Contexto de estudio: másteres en enseñanza de lenguas en el área de especialización de portugués y español u otra lengua extranjera en la Faculdade de Letras da Universidade do Porto}

El objetivo del presente trabajo es estudiar y analizar algunos de los intereses e inquietudes reflejados en los portafolios reflexivos de los profesores en prácticas (en adelante PeP) de los dos másteres oficiales en enseñanza de lenguas de la FLUP que tienen como opción el español (portugués como lengua materna o con otra lengua extranjera). Estos másteres, denominados en portugués, Mestrado em Ensino do Inglês e de Língua Estrangeira no 3ํㅜㄷo do Ensino Básico e do Ensino Secundário, nas áreas de especialização de Alemão ou Espanhol ou Francês (MEIEFA) ${ }^{2}$ y Mestrado em Ensino de Português e de Língua Estrangeira no 3o Ciclo do Ensino Básico e no Ensino Secundário nas áreas de especialização de Alemão ou Espanhol ou Francês ou Inglês $(\text { MEPIEFA })^{3}$, son segundos ciclos profesionalizantes, lo que en Portugal se entiende como un título oficial universitario que habilita para la enseñanza de lenguas en la educación obligatoria básica y secundaria 4 . Para completar estos másteres se deben obtener los 120 créditos ECTS de la especialidad prevista en el Decreto-Lei $n^{\circ}$ 43/2007, de 22 de Fevereiro, esto es, aprobar todas las unidades curriculares que integran sus planes de estudio y superar, en acto público, la defensa de un trabajo de fin de máster cuyo tema tiene que estar relacionado con la didáctica de las lenguas en las cuales se realizaron las prácticas supervisadas, tal y como se indica en el artículo 3 de sus reglamentos (FLUP, 2013, p.1). Asimismo, ambos másteres tienen como objetivo la adquisición de las competencias científicas y pedagógicas necesarias para el ejercicio de la función docente en enseñanza básica y secundaria, teniendo en cuenta las transformaciones de la sociedad, de la educación y de la evolución científica y tecnológica 5 .

\footnotetext{
2 Hasta el curso lectivo 2014/2015 se denominó MEPLE (Mestrado em Ensino do Português no $3^{\circ}$ Ciclo do Ensino Secundário e de Língua Estrangeira nos Ensinos Básico e Secundário).

${ }^{3}$ Hasta el curso lectivo 2014/2015 se denominó MEIBS (Mestrado em Ensino do Inglês e de Alemão/Francês/Espanhol no 3ํㅡㄴ Ciclo do Ensino Básico e Secundário).

${ }^{4}$ La educación básica en Portugal es obligatoria y comprende tres ciclos de nueve años en total entre los 6 y los 14 años de edad: el primer ciclo de cuatro años, el segundo de dos y el tercero de tres. El 3ำ ciclo do Ensino Básico (3. CEB) se corresponde con los tres primeros años de la Educación Secundaria Obligatoria (ESO) del sistema educativo español. En cuanto al Ensino Secundário en Portugal (ES), este consiste en un ciclo obligatorio (hasta los 18 años) de tres años que se corresponde con el último de la ESO y con los dos del Bachillerato en España.

${ }^{5}$ Información disponible sobre MEIEFA en la página oficial de la Faculdade de Letras da Universidade do Porto: https://bit.ly/2oXIPIJ
} 
Las unidades curriculares que han de cursarse en ambos másteres se distribuyen en dos años (cuatro semestres). En el primero, estas se centran en conocimientos teóricos de las áreas de Educación, Didáctica, Formación de profesores y Lenguas mientras que durante el segundo año la unidad curricular principal es Iniciação à Prática Profissional (IPP) puesto que con carácter anual reúne 48 de los 120 ECTS indicados anteriormente y se enmarca dentro del área científica de Formación de profesores, tal y como se prevé

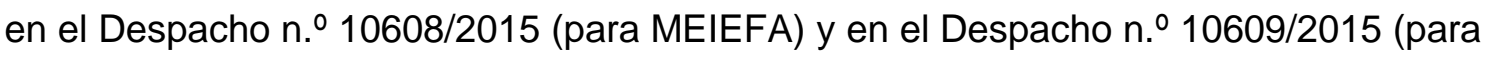
MEPIEFA) ${ }^{6}$. En ambos casos la IPP tiene cuatro componentes: unas prácticas formativas supervisadas (Prática de ensino supervisionada) acompañadas por un orientador del centro de enseñanza en el que tienen lugar y por un supervisor de la FLUP, un seminario de apoyo a la práctica profesional (Seminário), la elaboración de un trabajo de fin de máster (Relatório de estágio), que consiste en un proyecto de investigación-acción y su defensa pública. En este sentido, se debe resaltar la importancia dada, dentro de estos cursos, a la práctica reflexiva de los $\mathrm{PeP}$, tal y como se subraya en un documento interno donde también se indican las pautas para la elaboración de dicho trabajo:

A prática pedagógica supervisionada (estágio) tem como finalidade desenvolver a capacidade de reflexão do professor em formação inicial concebido como um profissional de ensino capaz de estabelecer relações entre a teoria e a prática. [...] O modelo reflexivo de formação de professores coloca um foco explícito na reflexão através da investigação de uma dimensão específica. Isto quer dizer que este modelo garante o desenvolvimento da reflexão e a prática reflexiva como dimensão crucial na prática de ensino e no ser professor. (Almeida et al., 2016, p. 5)

Para ello, se pone a disposición de los PeP que cursan MEIEFA y MEPIEFA, tal como recomiendan especialistas como Marta Higueras, «una serie de instrumentos para la reflexión sistemática, como portfolios, diarios, etc., que pueden ayudar al profesor en este proceso introspectivo y que deberían estar muy presentes en las actividades formativas» (2012, p. 110). En este sentido, además del proyecto de investigaciónacción anteriormente mencionado, en el reglamento de los másteres se considera como obligaciones de los PeP la elaboración de su portafolio de prácticas (portefólio de

\footnotetext{
${ }^{6}$ Las áreas científicas predominantes y los créditos que deben superarse para la obtención de los másteres (créditos que tienen todos carácter obligatorio) aparecen especificados en el Diário da República, 2. ${ }^{a}$ série - N. ${ }^{\circ} 186$ do 23 de setembro de 2015.
} 
estágio pedagógico) como soporte al proyecto de investigación. Se trata, por tanto, de un portafolio reflexivo de carácter obligatorio, según establecen los reglamentos de los másteres en enseñanza, y que tiene como objetivo principal fomentar la investigación, la reflexión y la capacidad de autoevaluación sobre la propia actuación docente. Estos fines van al encuentro de los objetivos generales de los másteres MEIEFA y MEPIEFA (por ejemplo, «Ao nível do desenvolvimento profissional ao longo da vida, fazer da formação inicial o ponto de partida de um itinerário alicerçado na reflexão sobre questões educativas e sobre a prática profissional»`7), así como al de los objetivos específicos de la asignatura Seminário de IPP («desenvolver capacidades e atitudes conducentes a um desempenho profissional reflexivo, problematizador, crítico e em permanente aperfeiçoamento» $y$ «analisar, reflexivamente, experiencias implementadas em cada núcleo de estágio $»^{8}$ ) y, por último, al de los objetivos específicos de las labores atribuidas a los orientadores que acompañan a los $\mathrm{PeP}$ durante sus prácticas, donde se destaca la necesidad de «promover a dimensão analítica, reflexiva e interpessoal da formacão inicial».

\section{3 - El portafolio docente como instrumento de reflexión. El caso concreto del portafolio de prácticas de español en MEIEFA y en MEPIEFA}

De los diversos instrumentos que facilitan al profesor, ya sea en formación inicial o no, justificar o aportar datos de su práctica, uno de ellos es el portafolio del docente que:

permite al profesor, por un lado, documentar de manera visible sus experiencias y resultados en la enseñanza, organizándolos de una forma personalizada. $Y$, por otro, a través de la reflexión, especificar y valorar qué tipo de actividades realiza, identificando problemas, sacando conclusiones y planteándose nuevas metas, lo cual contribuirá a esa mejora por la que nos preguntábamos. Esta última orientación es la que permite entroncar esta práctica, desde un enfoque constructivista, con la denominada investigación en acción (a medio camino entre la práctica docente reflexiva y la metodología de la investigación en adquisición de idiomas). (Pastor, 2012, p. 8)

\footnotetext{
7 Información disponible sobre MEIEFA en la página oficial de la Faculdade de Letras da Universidade do Porto: https://bit.ly/2oXIPIJ

${ }^{8}$ Los PeP se organizan en núcleos de prácticas (núcleos de estágio) constituidos por hasta tres profesores en formación inicial en una escuela pública cooperante con la facultad (FLUP, 2014, p. 9).
} 
En el caso concreto de nuestra investigación, el portafolio solicitado a los PeP de los másteres MEIEFA y MEPIEFA en el área de español no consiste en un compendio completo de todas las actividades docentes realizadas por ellos durante sus prácticas, ni en una muestra de las actividades realizadas por otros docentes, bien sean los compañeros del núcleo de prácticas, el orientador de la escuela o el supervisor de la facultad. Por el contrario, se trata de un portafolio reflexivo cuyo objetivo es servir de herramienta para la reflexión y autoevaluación de la competencia docente con base en el conjunto de conocimientos, habilidades y estrategias necesarias para enseñar idiomas, en nuestro caso español como lengua extranjera (ELE), a través de una muestra, basada en la selección de las decisiones pedagógicas tomadas durante el año en prácticas y la autoevaluación de su progreso, así como de la reformulación de sus ideas o creencias sobre la enseñanza-aprendizaje, aspectos todos ellos que en conjunto mostrarán su progreso como docente en formación inicial.

Este portafolio obligatorio, como ya se ha apuntado, se compone en el área de Español, de cuatro secciones:

- En la primera, el PeP debe hacer una breve descripción del centro educativo en el que realiza sus prácticas, del núcleo del que forma parte y de los grupos de alumnos a los que imparte clases de español.

- La segunda sección consiste en un análisis y reflexión personal de nueve descriptores de los $195^{9}$ que constituyen el Portfolio Europeo para futuros profesores de idiomas. Una herramienta de reflexión para la formación de profesores (PEFPI) (Newby et al., 2007) que los PeP seleccionan libremente de entre las siete categorías que lo conforman. No obstante, se recomienda a los PeP que escojan, en la medida de lo posible, descriptores relacionados con el tema de su trabajo de fin de máster con el objetivo de rentabilizar la información obtenida tras la reflexión sobre su práctica docente. El esquema que han de seguir para su realización es el siguiente: en el primer periodo de prácticas deben realizar la autoevaluación de tres descriptores; en el segundo la de dos nuevos descriptores, así como la de otro que ya se haya analizado en el primer periodo (señalando los posibles progresos realizados); y en el tercer periodo, la de dos nuevos descriptores y un tercero que ya haya sido comentado en los periodos anteriores especificando las mejoras alcanzadas.

\footnotetext{
${ }^{9}$ Se ha constatado que el número total de descriptores es 195 y no 193 , como se indica en la introducción del PEFPI (Newby et al., 2007, p. 5).
} 
- La tercera parte es también una autoevaluación que, a diferencia de la sección anterior, analiza de forma holística las clases impartidas sin partir de los descriptores del PEFPI que focalizan la atención en aspectos concretos. Esta autoevaluación está guiada por los tutores de la facultad que sugieren a los PeP ideas y preguntas específicas y además se les recomienda consultar los documentos institucionales ${ }^{10}$ analizados en el Seminário, a fin de fomentar y conducir la reflexión sobre su experiencia docente y evitar la descripción superficial.

- La cuarta y última sección del portafolio consiste en una conclusión en la que se recomienda volver a los objetivos marcados al inicio del año de prácticas y valorar en qué medida estos se han cumplido o no. También aquí se facilitan algunas preguntas que pueden resultar útiles a los PeP para hacer balance sobre sus prácticas docentes.

\section{4 - Descripción del estudio}

El objetivo del estudio aquí presentado es conocer las inquietudes de 60 profesores en prácticas inscritos entre 2014 y $2017^{11}$ en MEIEFA y MEPIEFA. Para ello, partimos del análisis de la segunda parte, antes referida, de su portafolio de prácticas (portefólio de estágio pedagógico), concretamente la sección en la que deben seleccionar nueve descriptores del PEFPI, dado que, como hemos explicado anteriormente, deben elegir tres después de cada uno de los tres periodos en torno a los que se estructuran las prácticas.

Antes de avanzar con la descripción de las fases de la investigación y la descripción de la muestra, conviene realizar una breve presentación del PEFPI para comprender la naturaleza de los datos obtenidos.

\section{1 - Portfolio Europeo para futuros profesores de idiomas. Una herramienta de reflexión para la formación de profesores (PEFPI)}

Este documento, desarrollado por el Consejo de Europa a través del Centro de Lenguas Modernas en el año 2007, constituye «un documento abierto y práctico dirigido

\footnotetext{
10 Nos referimos, además de al PEFPI, a Las Competencias clave del profesorado de lenguas segundas y extranjeras (Instituto Cervantes, 2012) y a la Parrilla del perfil del profesor de idiomas - EPG (VV. AA., 2013).

11 Si bien el PEFPI se viene utilizando en nuestro contexto de formación inicial de profesores de español desde el curso 2010-2011.
} 
a los profesores europeos de idiomas que están en un primer estadio de formación » ${ }^{12}$ (Verdía, 2016, p. 42) y está concebido para reflexionar sobre las destrezas y conocimientos didácticos necesarios para enseñar idiomas, ayudar a evaluar competencias didácticas, permitirnos seguir el progreso y registrar experiencias como profesores en el transcurso de la formación inicial (Newby et al., 2007, p. 5).

Los objetivos generales de este portafolio son: fomentar reflexiones sobre las competencias que un profesor se esfuerza en conseguir y en el conocimiento subyacente que alimenta esas competencias; ayudar a prepararlo para su futura profesión en una variedad de contextos de aprendizaje; promover la discusión con sus compañeros y con sus profesores tutores; facilitar la autoevaluación del desarrollo y adquisición de sus competencias; y ofrecer una herramienta que le ayude a visualizar su progreso (Newby et al., 2007, p. 5).

En cuanto a sus contenidos, el PEFPI consta de seis secciones: una declaración personal que tiene como objetivo reflexionar sobre cuestiones generales relacionadas con la enseñanza; una sección de autoevaluación de la competencia docente que contiene 195 descriptores (entendidos como el conjunto de competencias básicas que todo profesor de idiomas debería esforzarse en desarrollar y adquirir) formulados en términos de capacidad "sé o puedo hacer», junto al que aparece una barra que sirve para visualizar y registrar distintos momentos del desarrollo de su competencia; un dossier que ayuda a evidenciar de forma transparente los resultados de su autoevaluación, mostrar el progreso y registrar ejemplos de su trabajo; un glosario de términos relacionados con la enseñanza y aprendizaje de idiomas; un índice de términos utilizados en los descriptores; y una guía de utilización sobre este documento (Newby et al., 2007, p. 5).

Sin embargo, la parte central del PEFPI, y en la cual nos centraremos para la recogida de datos, es la sección de autoevaluación donde los descriptores se agrupan en siete categorías generales: «Contexto», «Metodología», «Recursos», «Programación del curso», «Dar clase», «Aprendizaje autónomo» y «Evaluación del aprendizaje». Estas categorías «representan áreas en las que los profesores necesitan tener conocimientos y haber desarrollado distintas competencias esenciales y en las que deben tomar decisiones en relación con la enseñanza» (Newby et al., 2007, p. 6). Cada una de estas categorías se subdivide en diferentes áreas y cada área en varios

12 Aunque consideramos que como herramienta de reflexión para la autoevaluación de la competencia docente no tiene por qué restringirse a la formación inicial, sino que puede aplicarse en cualquier momento del desarrollo profesional de un profesor de lenguas. 
BARROS LORENZO, Mónica; Nicolás Martínez, Pilar; Pazos Anido, Marta - Aproximación a los intereses e inquietudes de los profesores... Para lá da tarefa: implicar os estudantes na aprendizagem de línguas estrangeiras no ensino superior. Porto: FLUP, 2019, pp. 44-67 DOI: https://doi.org/10.21747/9789898969217/paraa3

descriptores. Por ejemplo, la categoría «Dar clase», a la que nos referiremos más adelante, se divide en cinco áreas: «Uso de la programación», «Contenidos», «Interacción con los alumnos», «Dinámicas y gestión de la clase» y «La lengua de la clase»; y estas a su vez en descriptores, siendo el primero de ellos, a modo de ejemplo: «Sé comenzar una clase transmitiendo motivación» (Newby et al., 2007, p. 41), correspondiente al área «Uso de la programación».

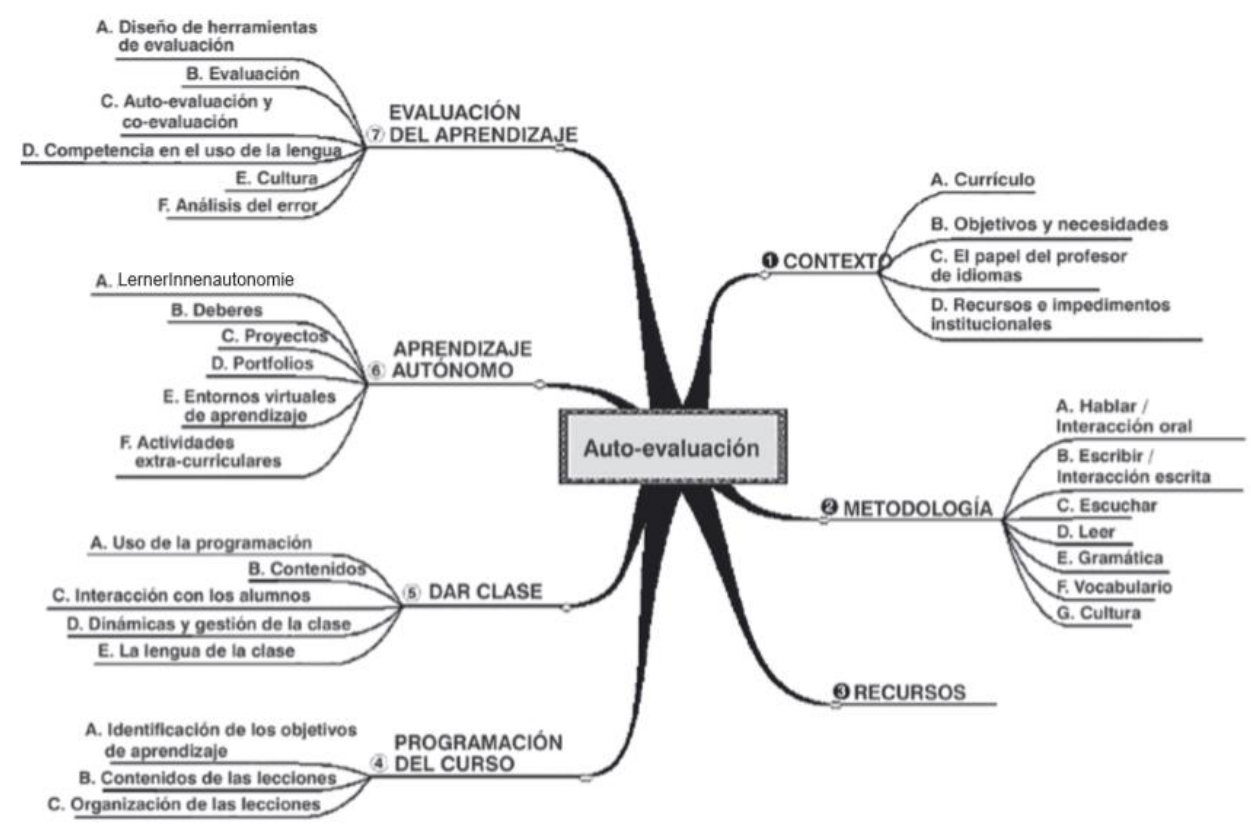

Figura 2. Categorización de los descriptores del Portfolio Europeo para futuros profesores de idiomas. (PEFPI). (Newby et al., 2007, p. 6).

\section{2 - Fases del estudio}

Para una descripción más pormenorizada del estudio seguimos las fases de investigación cualitativa propuestas por José Ignacio Ruiz Olabuénaga (2007, p. 51): el diseño del trabajo, la recogida de datos, el análisis de los mismos y la elaboración del informe.

Como ya hemos anticipado, nuestra finalidad es conocer las inquietudes de los PeP inscritos en los programas de formación inicial MEIEFA y MEPIEFA a partir de la selección que realizan de los 195 descriptores del PEFPI para apoyar su reflexión. No se define, por lo tanto, un problema como tal ni se parte de una determinada hipótesis, pues como advierte Marta Sabariego (2004, p. 129), no siempre es necesario, ya que los métodos cualitativos con una finalidad descriptiva o exploratoria tienen un carácter inductivo y, por tanto, no se suele partir de hipótesis antes de la recogida y análisis de los datos. El objetivo es, en este primer estadio de la investigación, obtener datos 
BARROS LORENZO, Mónica; Nicolás Martínez, Pilar; Pazos Anido, Marta - Aproximación a los intereses e inquietudes de los profesores... Para lá da tarefa: implicar os estudantes na aprendizagem de línguas estrangeiras no ensino superior. Porto: FLUP, 2019, pp. 44-67 DOI: https://doi.org/10.21747/9789898969217/paraa3

precisos acerca de los descriptores que seleccionan los PeP en el contexto de enseñanza básica y secundaria en Portugal a fin de obtener algunas consideraciones que puedan tener implicaciones para un análisis posterior más profundo de los datos y, en última instancia, para una reflexión sobre las posibles causas y las consecuencias de esta selección en la formación inicial de profesores del área de especialización de Español de MEIEFA y MEPIEFA.

Una vez definido el objetivo, siguiendo los pasos del diseño del estudio, se decide cuál será la muestra objeto de análisis. En este caso, se parte de 60 documentos escritos, concretamente, de los portafolios de prácticas de 60 estudiantes, de los cuales 22 tienen experiencia previa en la enseñanza de lenguas extranjeras y 38 no, todos ellos inscritos en el segundo año de los ciclos de estudio MEIEFA y MEPIEFA de la Universidade do Porto en los cursos lectivos 2014-2015, 2015-2016 y 2016-2017, años en los que realizaron sus prácticas pedagógicas en distintos centros educativos públicos portugueses del tercer ciclo de la enseñanza básica y en secundaria, situados en Oporto y sus alrededores. De esta manera, optamos por una técnica de muestreo no probabilístico (Ruiz Olabuénaga, 2007, p. 64; Sabariego, 2004, p. 147), pues la selección de los participantes, o en este caso, de los portafolios, se realiza de manera «deliberada e intencional» (Rodríguez, Gil \& García, 1996, p. 135), siendo los principales criterios la disponibilidad y el acceso a la consulta de los mismos. Partimos, de esta manera, en este estadio inicial de estudio, del portafolio de prácticas como única fuente de datos, que, por tratarse de un documento escrito, como considera Creswell (2014, pp. 191-192), facilita el acceso en cualquier lugar y momento, y cuenta con la gran ventaja de que no precisa transcripción; si bien existe el riesgo de que algún documento puede estar incompleto o no ser preciso, en nuestro caso, sesgos fácilmente evitables con la revisión exhaustiva de los textos.

De este modo, para la recogida de datos recurrimos al análisis documental, una de las estrategias de obtención de información propuesta por Del Rincón, Argal, Latorre y Sans (1995, p. 37). De los documentos analizados, en esta primera fase del estudio, nos basamos en una investigación exploratoria de los descriptores seleccionados (Sabariego \& Bisquerra, 2004, p. 95), centrándonos únicamente en los descriptores, sin realizar el estudio de la reflexión de cada uno de ellos. Por esto, a pesar de que se trata de una investigación cualitativa basada en evidencia escrita y de que la técnica de análisis consiste en el análisis de contenido (Sabariego, Dorio \& Massot, 2004, p. 302), si se tiene en cuenta el objetivo inicial de este estudio, el análisis de los datos, en este caso, es más cuantitativo. Como admite Ruiz Olabuénaga (2007, p. 232), no existe un 
BARROS LORENZO, Mónica; Nicolás Martínez, Pilar; Pazos Anido, Marta - Aproximación a los intereses e inquietudes de los profesores... Para lá da tarefa: implicar os estudantes na aprendizagem de línguas estrangeiras no ensino superior. Porto: FLUP, 2019, pp. 44-67 DOI: https://doi.org/10.21747/9789898969217/paraa3

único modelo de análisis de los datos, sino que depende de los intereses, los objetivos, las condiciones y los textos y, aunque pueda parecer antagónico, como explican Rodríguez, Gil y García: «la cuantificación es un aspecto considerado por muchos de los autores que se enmarcan en líneas de investigación cualitativas» (1996, p. 216). De hecho, tal como afirma Ruiz Olabuénaga, uno de los enfoques tradicionales del análisis de texto está asociado al análisis cuantitativo (2007, p. 194). En esta misma línea, Del Rincón et al. reconocen que, a pesar del carácter cualitativo de ciertos datos, es posible analizarlos de manera cuantitativa, sobre todo, como es este caso, cuando pretendemos ver la frecuencia, es decir, «el número de veces que ocurre una determinada categoría» (1995, p. 242), pues ese recuento cuantitativo de las ocurrencias de una determinada palabra o fragmento puede resultar muy útil cuando el cuerpo de datos es muy amplio o cuando nos interesa conocer precisamente ese dato acerca del número de veces de una repetición. Si bien este análisis cuantitativo de datos cualitativos nos permite asegurar con mayor facilidad la fiabilidad y la objetividad de la información, también es cierto que resulta bastante reductor y desaprovecha la riqueza que puede aportar un texto si se explora con otras técnicas, por lo que lo ideal es que se sigan otras técnicas cualitativas que profundicen en el contenido (Rodríguez, Gil \& García, 1996, pp. 217218; Ruiz Olabuénaga, 2007, p. 234), tal como se procederá en trabajos futuros tras este primer acercamiento a los datos del que se da cuenta en este artículo.

En los datos obtenidos para el análisis que aquí se presenta nos centraremos únicamente en la frecuencia, es decir, en el número de veces que los PeP seleccionan un descriptor. Por lo tanto, teniendo en cuenta el objetivo de esa fase del estudio, se requiere un tratamiento cuantitativo de los datos y, para ello, nos basamos en las fases de los procesos aplicados en el análisis cuantitativo propuestas por Joan Mateo y Francesc Martínez (2008, p. 41). En primer lugar, procedemos a la codificación de los descriptores del PEFPI, numerándolos del 1 al 195 a fin de facilitar la recogida de los datos incluidos en los portafolios. Después, creamos una matriz en una hoja Excel donde incluimos los datos de los autores de los portafolios, indicando el curso académico en el que están realizando las prácticas docentes, si tienen experiencia docente previa o no y los 9 descriptores seleccionados. A continuación, decidimos las pruebas estadísticas que vamos a aplicar a fin de determinar los descriptores escogidos más veces por los PeP y a qué categorías, de las siete incluidas en el PEFPI, corresponden. No obstante, no basta con calcular la frecuencia, sino que, dado que no todas las categorías cuentan con el mismo número de áreas ni de descriptores,es necesario hallar también la ponderación, es decir, el peso de cada una de las categorías 
BARROS LORENZO, Mónica; Nicolás Martínez, Pilar; Pazos Anido, Marta - Aproximación a los intereses e inquietudes de los profesores... Para lá da tarefa: implicar os estudantes na aprendizagem de línguas estrangeiras no ensino superior. Porto: FLUP, 2019, pp. 44-67 DOI: https://doi.org/10.21747/9789898969217/paraa3

del PEFPI con respecto a su conjunto (véase la explicación de la nota 13). Una vez decididas las pruebas necesarias, se implementan y se procede al análisis de los datos obtenidos y a la interpretación de los resultados, procesos descritos en los dos apartados siguientes.

\section{5 - Análisis de los datos}

Como acabamos de explicar, antes de pasar al cálculo de la frecuencia de los descriptores del PEFPI seleccionados más veces por los PeP, es importante tener en cuenta el número total de descriptores de cada una de las categorías y su porcentaje respecto al conjunto de los 195 descriptores:

\begin{tabular}{|l|c|c|}
\hline Categorias del PEFPI & $\begin{array}{l}\mathbf{N}^{0} \text { de descriptores del } \\
\text { PEFPI en cada categoria }\end{array}$ & $\begin{array}{l}\text { Porcentaje de cada categoria } \\
\text { en el PEFPI (factor A) }\end{array}$ \\
\hline 1. Contexto & 23 & $11,8 \%$ \\
\hline 2. Metodología & 57 & $29,2 \%$ \\
\hline 3. Recursos & 11 & $5,64 \%$ \\
\hline 4. $\begin{array}{l}\text { Programación del } \\
\text { curso }\end{array}$ & 22 & $11,3 \%$ \\
\hline 5. Dar clase & 27 & $13,8 \%$ \\
\hline 6. Aprendizaje autónomo & 28 & $14,4 \%$ \\
\hline 7. $\begin{array}{l}\text { Evaluación del } \\
\text { aprendizaje }\end{array}$ & 27 & $13,8 \%$ \\
\hline Total & $\mathbf{1 9 5}$ & $\mathbf{1 0 0} \%$ \\
\hline
\end{tabular}

Tabla 1. Categorías del PEFPI, número de descriptores por categoría y porcentaje de cada una con respecto a su conjunto.

Centrándonos ahora en los datos recogidos en nuestro estudio, conviene destacar que se obtienen un total de 540 respuestas, pues se analizan los portafolios de $60 \mathrm{PeP}$ $y$, como ya se ha explicado, cada uno de ellos selecciona 9 descriptores, de los cuales siete son en todos los casos diferentes y uno o dos de ellos se retoma en la reflexión posterior al segundo y tercer periodo de las prácticas. 


\begin{tabular}{|c|c|c|c|}
\hline $\begin{array}{l}\text { Categorias del } \\
\text { PEFPI }\end{array}$ & $\begin{array}{l}\mathrm{N}^{0} \text { de descriptores } \\
\text { escogidos por los } \mathrm{PeP} \\
\text { en cada categoría para } \\
\text { esta muestra }\end{array}$ & $\begin{array}{l}\text { Porcentaje por categoría } \\
\text { según los descriptores } \\
\text { escogidos por los PeP } \\
\text { (factor B) }\end{array}$ & $\begin{array}{l}\text { Ponderación } \\
\text { de los } \\
\text { factores A y } \\
\mathbf{B}^{13}\end{array}$ \\
\hline 1. Contexto & 83 & $15,4 \%$ & 1,30 \\
\hline 2. Metodología & 137 & $25,4 \%$ & 0,87 \\
\hline 3. Recursos & 60 & $11,1 \%$ & 1,97 \\
\hline $\begin{array}{l}\text { 4. Programación } \\
\text { del curso }\end{array}$ & 62 & $11,5 \%$ & 1,02 \\
\hline 5. Dar clase & 103 & $19,1 \%$ & 1,38 \\
\hline $\begin{array}{l}\text { 6. Aprendizaje } \\
\text { autónomo }\end{array}$ & 43 & $7,96 \%$ & 0,55 \\
\hline $\begin{array}{l}\text { 7. Evaluación del } \\
\text { aprendizaje }\end{array}$ & 52 & $9,63 \%$ & 0,70 \\
\hline Total & 540 & $100 \%$ & Promedio: 1 \\
\hline
\end{tabular}

Tabla 2. Descriptores del PEFPI seleccionados por los PeP por cada categoría, porcentaje por categoría según los descriptores seleccionados y ponderación de los factores A y B, según se explica en la nota 13.

Tomando en consideración el porcentaje que tiene cada categoría en el conjunto del PEFPI y el porcentaje por categoría que se obtiene del número de descriptores escogidos por los PeP se concluye, al realizar la ponderación ${ }^{13}$, que la categoría a la que corresponden los descriptores más seleccionados es «Recursos».

No obstante, considerando que casi la mitad de las veces (46\%) se elige el mismo descriptor (es decir, de las 83 ocurrencias dentro de la categoría «Recursos», 38 hacen referencia al mismo ${ }^{14}$ ), decidimos optar — teniendo en cuenta el cálculo anterior relativo a la ponderación-, por la segunda categoría más seleccionada «Dar clase», dado que consta de una mayor variedad de descriptores elegidos.

Antes de continuar con el análisis, conviene describir brevemente en qué consiste esta categoría. De las siete categorías generales del PEFPI el área «Dar clase» se centra en «cómo llevar a cabo una clase de lengua moderna, trata de qué hace un profesor de lengua moderna en clase y de las destrezas necesarias que ha de poner en juego» (Newby et al., 2007, p. 40). Esta categoría, como indicamos anteriormente, se divide en cinco áreas. En la primera, «Uso de la programación», los descriptores se

${ }_{13}$ Para poder evaluar la importancia que cada categoría del PEFPI tiene para los PeP definimos un factor de ponderación obtenido de la división del porcentaje por categoría según los descriptores escogidos por los PeP (factor B) entre el porcentaje que tiene cada categoría en el conjunto del PEFPI (factor A).

${ }^{14}$ Este es: «Sé diseñar materiales y actividades de aprendizaje apropiadas para mis alumnos». 
BARROS LORENZO, Mónica; Nicolás Martínez, Pilar; Pazos Anido, Marta - Aproximación a los intereses e inquietudes de los profesores... Para lá da tarefa: implicar os estudantes na aprendizagem de línguas estrangeiras no ensino superior. Porto: FLUP, 2019, pp. 44-67 DOI: https://doi.org/10.21747/9789898969217/paraa3

refieren a cómo planificar una unidad didáctica (secuenciación de actividades, temporalización, motivación, flexibilización, reajustes, etc.). En la segunda, «Contenidos», hacen hincapié en la presentación de los contenidos lingüísticos de forma apropiada, en su relación con lo que ya conocen los estudiantes, con temas de actualidad y con la cultura de la lengua que están aprendiendo. En la tercera, «Interacción con los alumnos», se centran en reflexionar sobre la importancia de captar y mantener la atención de los alumnos, apoyar y motivar sus participaciones e iniciativas, tener en cuenta los distintos estilos de aprendizaje, así como en ayudarles a desarrollar estrategias de aprendizaje. En la cuarta, «Dinámicas y gestión de clase», ponen énfasis en los distintos papeles del profesor en el aula, en la gestión de distintas formas de trabajo, la eficacia de recursos pedagógicos y en cómo trabajar y asistir a los alumnos en el uso de las TIC (tecnologías de la información y la comunicación). Por último, en la quinta, «La lengua de la clase», se tiene en cuenta la capacidad del profesor de dar la clase en la lengua meta, su uso como metalenguaje, cuándo usarla, cómo motivar a los estudiantes para utilizarla, así como en qué manera relacionarla con otras lenguas que conocen (Newby et al., 2007, pp. 40-45).

A continuación, presentamos una tabla con los resultados obtenidos para la categoría «Dar clase», formada por 5 áreas y 27 descriptores, con la indicación del número de veces que han sido seleccionados los descriptores correspondientes a cada una de estas áreas, así como el peso de cada una de ellas respecto al número total de descriptores de dicha categoría.

\begin{tabular}{|l|c|c|c|}
\hline Categoría «Dar clase»" & $\begin{array}{l}\text { Cantidad de veces } \\
\text { que los PeP escogen } \\
\text { descriptores relativos } \\
\text { a cada área de la } \\
\text { categoría «Dar clase» }\end{array}$ & $\begin{array}{l}\text { Porcentaje de } \\
\text { cada área según } \\
\text { lo seleccionado } \\
\text { por los PeP }\end{array}$ & $\begin{array}{l}\text { Relación con el } \\
\text { porcentaje de } \\
\text { cada área en el } \\
\text { PEFPI }\end{array}$ \\
\hline $\begin{array}{l}\text { A. Uso de la } \\
\text { programación }\end{array}$ & 41 & $40 \%$ & $22 \%$ \\
\hline B. Contenidos & 7 & $7 \%$ & $15 \%$ \\
\hline $\begin{array}{l}\text { C. Interacción con los } \\
\text { alumnos }\end{array}$ & 22 & $21 \%$ & $22 \%$ \\
\hline $\begin{array}{l}\text { D. Dinámicas y gestión } \\
\text { de la clase }\end{array}$ & 16 & $16 \%$ & $19 \%$ \\
\hline E. La lengua de la clase & 17 & $17 \%$ & $22 \%$ \\
\hline Total & $\mathbf{1 0 3}$ & $\mathbf{1 0 0} \%$ & $\mathbf{1 0 0} \%$ \\
\hline
\end{tabular}


BARROS LORENZO, Mónica; Nicolás Martínez, Pilar; Pazos Anido, Marta - Aproximación a los intereses e inquietudes de los profesores... Para lá da tarefa: implicar os estudantes na aprendizagem de línguas estrangeiras no ensino superior. Porto: FLUP, 2019, pp. 44-67 DOI: https://doi.org/10.21747/9789898969217/paraa3

Tabla 3. Frecuencia con la que los PeP escogen descriptores relativos a las cinco áreas de la categoría «Dar clase» del PEFPI, porcentaje de cada una de estas áreas en función de los descriptores seleccionados y porcentaje de las áreas según el número total de descriptores de cada una de ellas.

En la tabla anterior observamos que en la categoría «Dar clase» el área que despierta un mayor interés entre los $60 \mathrm{PeP}$ (con un 40\% de descriptores seleccionados) es «Uso de la programación», de acuerdo con el número de veces que los PeP optan por descriptores de esa categoría (40\%); área que, en cambio, no ostenta un lugar destacado en el porcentaje global de esta categoría, pues las cinco que la componen tienen un número muy similar de descriptores (entre 6 y 4). La siguiente preocupación está relacionada con la «Interacción con los alumnos», elegida el $21 \%$ de las veces. Seguidamente, y con valores muy parecidos, las áreas de «La lengua de la clase» y «Dinámicas y gestión de la clase», con un 17\% y 16\% respectivamente. Por último, decae el interés a un 7\% el área de «Contenidos».

Además de estos datos generales, interesa conocer los descriptores seleccionados con mayor frecuencia de los 27 que conforman la categoría «Dar clase». De todos ellos apuntamos los cinco elegidos con mayor frecuencia, por orden descendente, por parte de los $38 \mathrm{PeP}$ sin experiencia docente previa al momento de realización de las prácticas y de los $22 \mathrm{PeP}$, con la indicación del número de veces que son seleccionados por los PeP y si en algún caso son retomados en un segundo o tercer momento durante el periodo de prácticas. 
BARROS LORENZO, Mónica; Nicolás Martínez, Pilar; Pazos Anido, Marta - Aproximación a los intereses e inquietudes de los profesores... Para lá da tarefa: implicar os estudantes na aprendizagem de línguas estrangeiras no ensino superior. Porto: FLUP, 2019, pp. 44-67 DOI: https://doi.org/10.21747/9789898969217/paraa3

\begin{tabular}{|c|c|c|c|}
\hline \multicolumn{4}{|c|}{ CATEGORIÁ: «DAR CLASE» } \\
\hline AREAS & DESCRIPTOR & $\begin{array}{l}\text { PePCON } \\
\text { EXPERIENCIA }\end{array}$ & $\begin{array}{l}\text { PePSIN } \\
\text { EXPERIENCIA }\end{array}$ \\
\hline \multirow[b]{2}{*}{$\begin{array}{l}\text { Uso de la } \\
\text { programación }\end{array}$} & \multirow{2}{*}{$\begin{array}{l}\mathrm{N}^{0} 116^{15} \\
\text { Soy capaz de asegurar una } \\
\text { transición natural entre } \\
\text { actividades y tareas realizadas } \\
\text { individualmente, en grupos, o } \\
\text { entre toda la clase. }\end{array}$} & \multicolumn{2}{|c|}{ Número de veces seleccionado: 18} \\
\hline & & $\begin{array}{l}4 \text { (retomado } 2 \\
\text { veces) }\end{array}$ & $\begin{array}{l}14 \text { (retomado } 6 \\
\text { veces) }\end{array}$ \\
\hline \multirow[b]{2}{*}{$\begin{array}{l}\text { Uso de la } \\
\text { programación }\end{array}$} & \multirow{2}{*}{$\begin{array}{l}\mathrm{N}^{0} 114 \\
\text { Sé comenzar una clase } \\
\text { transmitiendo motivación. }\end{array}$} & \multicolumn{2}{|c|}{ Número de veces seleccionado: 12} \\
\hline & & $\begin{array}{l}8 \text { (retomado } 4 \\
\text { veces) }\end{array}$ & 4 \\
\hline \multirow[b]{2}{*}{$\begin{array}{l}\text { La lengua de } \\
\text { la clase }\end{array}$} & \multirow{2}{*}{$\begin{array}{l}N^{0} 135 \\
\text { Soy capaz de dar la clase en } \\
\text { la lengua objeto de estudio } \\
\text { (lenqua meta). }\end{array}$} & \multicolumn{2}{|c|}{ Número de veces seleccionado: 9} \\
\hline & & ( & 7 (retomado $1 \mathrm{vez}$ ) \\
\hline \multirow{2}{*}{$\begin{array}{l}\text { Interacción } \\
\text { con los } \\
\text { alumnos }\end{array}$} & \multirow{2}{*}{$\begin{array}{l}\text { N. }{ }^{0} 127 \\
\text { Sé motivar la participación de } \\
\text { los alumnos siempre que sea } \\
\text { posible. }\end{array}$} & \multicolumn{2}{|c|}{ Número de veces seleccionado: 8} \\
\hline & & 2 (retomado $1 \mathrm{vez}$ ) & $\begin{array}{l}6 \text { (retomado } 2 \\
\text { veces) }\end{array}$ \\
\hline \multirow[b]{2}{*}{$\begin{array}{l}\text { Dinámicas y } \\
\text { gestión de la } \\
\text { clase }\end{array}$} & \multirow{2}{*}{$\begin{array}{l}\text { N. }{ }^{0} 131 \\
\text { Sé crear, gestionar y } \\
\text { rentabilizar las ocasiones para } \\
\text { trabajar de manera individual, } \\
\text { en parejas, en grupos o toda } \\
\text { la clase. }\end{array}$} & \multicolumn{2}{|c|}{ Número de veces seleccionado: 8} \\
\hline & & 2 (retomado $1 \mathrm{vez}$ ) & $\begin{array}{l}6 \text { (retomado } 2 \\
\text { veces) }\end{array}$ \\
\hline
\end{tabular}

Tabla 4. Descriptores de la categoría «Dar clase» seleccionados con más frecuencia por los PeP con o sin experiencia docente previa con indicación del número de veces retomado en más de un periodo de las prácticas. ${ }^{15}$

La tabla anterior nos muestra los cinco descriptores más seleccionados por parte de los $60 \mathrm{PeP}$ cuyos portafolios de prácticas examinamos en este estudio y además nos permite observar el área a la que pertenecen dentro de la categoría «Dar clase», las frecuencias en función de si los PeP tienen o no experiencia docente previa a las prácticas y si el descriptor es escogido en uno o en más de uno de los tres periodos en torno a los que se estructuran las prácticas. Desglosemos estos datos para profundizar en este análisis.

Como ya se ha comentado, los dos descriptores seleccionados con más frecuencia, como se puede observar en la tabla, corresponden al área «Uso de la programación» de la categoría «Dar clase» y, de los cinco, también son los que más veces retoman los PeP para volver a reflexionar sobre ellos a lo largo de su periodo de prácticas docentes. El primero ( $n^{\circ}$ 116) hace referencia a la capacidad para realizar una transición natural entre actividades y, de las 18 veces que es seleccionado, 14 de ellas lo hacen profesores

${ }^{15}$ Recordamos que la numeración utilizada corresponde a la codificación de los descriptores del $P E F P I$ realizada por las autoras y no a la usada en el documento original. 
sin experiencia docente previa a las prácticas. Sin embargo, el segundo descriptor (no $114)$, relacionado con la capacidad de motivar al alumnado al inicio de la clase, de las 12 ocurrencias registradas, 8 de ellas corresponden a PeP con experiencia docente previa y son estos los únicos que reflexionan sobre este descriptor más de una vez, siendo este el único de los cinco descriptores de la tabla en los que se observan estas características. En cuanto al tercero (n 135), el cual versa sobre la capacidad de impartir la clase en la lengua meta y corresponde al área «La lengua de la clase», es seleccionado con mayor frecuencia por parte de PeP sin experiencia docente, en concreto 7 de las 9 veces, y de estas 9 veces en las que lo escogen, solo uno de ellos lo retoma en más de una ocasión. Respecto a los dos últimos descriptores, tanto el no 127, - perteneciente al área «Interacción con los alumnos», concretamente la capacidad de motivar la participación del alumnado-, como el no 131, correspondiente al área «Dinámicas y gestión de la clase» y relacionado con el saber crear, gestionar y rentabilizar las oportunidades para trabajar con distintos tipos de agrupamiento-, los datos registrados son idénticos. Ambos descriptores son seleccionados en 8 ocasiones, 6 por parte de PeP sin experiencia docente previa y 2 por PeP con experiencia docente y además son retomados en más de un periodo, específicamente en una ocasión por los PeP con experiencia docente y en dos por los que no la tienen.

En definitiva, constatamos que los cinco descriptores corresponden a cuatro de las cinco áreas que integran la categoría «Dar clase» en el $P E F P I$, de manera que la única que no se encuentra representada en estos primeros puestos es el área «Contenidos», y los dos seleccionados con mayor frecuencia pertenecen al área «Uso de la programación». Por otro lado, son los PeP sin experiencia docente previa a las prácticas los que optan en más ocasiones por estos descriptores, excepto el no 114 («Sé comenzar una clase transmitiendo motivación»), que registra el doble de ocurrencias entre los PeP con experiencia docente.

\section{6 - Consideraciones finales}

Para terminar, es conveniente trazar relaciones entre las conclusiones extraídas por otros investigadores en los estudios expuestos en la introducción de este trabajo y los datos obtenidos tras nuestra investigación.

Por ejemplo, la primera preocupación docente que señala Veenman (1984, pp. 154$155)$ en los problemas detectados como más frecuentes entre los profesores principiantes (ver Figura 1), se menciona la «Disciplina en la clase», asunto que no se 
BARROS LORENZO, Mónica; Nicolás Martínez, Pilar; Pazos Anido, Marta - Aproximación a los intereses e inquietudes de los profesores... Para lá da tarefa: implicar os estudantes na aprendizagem de línguas estrangeiras no ensino superior. Porto: FLUP, 2019, pp. 44-67 DOI: https://doi.org/10.21747/9789898969217/paraa3

destaca en los portafolios reflexivos de los PeP. No obstante, los profesores en formación inicial admiten verbalmente que les preocupa este tema pensando en un futuro cercano cuando, en los siguientes años como docentes principiantes, vayan a asumir la total responsabilidad de sus grupos. Pues, se debe tener en cuenta que en las clases que imparten los PeP durante su periodo en prácticas están presentes en el aula el orientador o la orientadora de la escuela (profesor responsable del grupo de alumnos), el supervisor o supervisora de la FLUP y los otros compañeros en prácticas que forman parte del núcleo de estágio. En este contexto se suele confirmar que (salvo casos muy puntuales) los alumnos adolescentes no demuestran indisciplina, por el contrario, tienden a ser colaborativos con los PeP durante sus clases observadas.

Con respecto al segundo problema más frecuente detectado en la ya mencionada lista de Veenman, relativo a «Motivar a los estudiantes», coincide con el asunto principal de dos de los descriptores del PEFPI más veces seleccionados por los PeP dentro de la categoría «Dar clase», estos son, el № 114 (en segundo lugar) y el no 127 (en cuarta posición). En el descriptor no 114 el foco se sitúa en el hecho de comenzar la clase trasmitiendo motivación, algo que interesa más a los PeP con experiencia, ya conscientes de la importancia de captar la atención de sus estudiantes desde un principio para que la clase se desarrolle de manera más fluida. En cambio, los PeP sin experiencia están más interesados en lograr motivar la participación de sus alumnos en la clase. De hecho, podría entenderse este matiz de diferencia a la luz de las tres etapas señaladas por Fuller (1969) por las que pasan las preocupaciones de los docentes. Por ello, considerando que en la primera etapa las inquietudes que preocupan a los profesores se centran en sí mismos, parece lógico que los PeP sin experiencia seleccionen con más frecuencia un descriptor relacionado con la motivación dentro del área «Interacción con los alumnos» y, en cambio, los PeP con experiencia estén más interesados en escoger otro concerniente a la motivación dentro del área «Uso de la programación», aspecto más en consonancia con la segunda etapa descrita por Fuller en la que prevalece la preocupación sobre las tareas docentes.

El tercer descriptor más seleccionado por los PeP es el no 135 dentro del área «La lengua de la clase» que plantea la posibilidad de impartir una clase en la lengua meta. Es comprensible la preocupación de los PeP sobre la propia competencia lingüística en español, dado que debe tenerse en cuenta que no tienen el español como lengua materna o primera, pese a que para acceder al máster tengan que acreditar un nivel B2.2 o C1.1 (dependiendo de la lengua extranjera que vayan a impartir). En concreto de las 9 veces que fue tratado este ítem en los portafolios, 7 de ellas fue escogido por 
BARROS LORENZO, Mónica; Nicolás Martínez, Pilar; Pazos Anido, Marta - Aproximación a los intereses e inquietudes de los profesores... Para lá da tarefa: implicar os estudantes na aprendizagem de línguas estrangeiras no ensino superior. Porto: FLUP, 2019, pp. 44-67 DOI: https://doi.org/10.21747/9789898969217/paraa3

profesores sin experiencia a los cuales es probable que les inquiete el poder conciliar, durante una clase completa, el control y corrección de su producción oral en español con la interacción con los alumnos y la gestión de clase; tareas simultáneas que los PeP con experiencia pueden tener más interiorizadas.

El quinto descriptor que han elegido los PeP de manera más reiterada, preponderantemente los que no tienen experiencia, es el no 131 localizado dentro del área «Dinámicas y gestión de la clase», que mueve a la reflexión docente sobre aspectos concernientes a como crear, gestionar y rentabilizar las diferentes agrupaciones en el aula, con la intención de variar los modos de trabajo. Este ítem podría, a grandes rasgos, relacionarse con el problema identificado por Veenman en sexto lugar: «Organización del trabajo en clase».

Con todo, y a pesar de que se observan contrastes entre las elecciones de los PeP con o sin experiencia docente previa, verificamos en nuestro estudio algo que ya había apreciado Veenman: que las diferencias entre los intereses e inquietudes de ambos tipos de PeP no son en exceso significativas. Si en vez de analizar los resultados en términos de descriptores, lo hacemos centrándonos en las áreas que componen la categoría «Dar clase», se advierte que de las 5 los PeP escogen descriptores mayoritariamente del área «Uso de la programación» (un 40\%), seguido de «Interacción con los alumnos» (un 21\%), mostrando todos ellos un escaso interés por el área “Contenidos» (un 7\%), pues revelan tener otras prioridades a la presentación adecuada de los contenidos al alumnado y a la vinculación de estos con sus conocimientos previos o con otros temas de actualidad. Las etapas de Fuller (1969) anteriormente comentadas o las directrices relativas a los contenidos de un curso determinados previamente por los documentos normativos o reguladores de la Direção-Geral da Educação (Aprendizagens Essenciais, Programas homologados para o 3. ciclo y Programas para o Ensino Secundário) y otros recursos como el libro de texto explican tal vez esta falta de interés.

Centrándonos en el contexto educativo portugués, Maria Assunção Flores (1999, p. 172) señaló los escasos estudios realizados en Portugal con respecto a este tema y apuntaba a que las principales preocupaciones de los docentes principiantes recaían en aspectos de naturaleza situacional y personal (concurrentes con similares investigaciones internacionales). Además de resaltar que las dificultades más recalcadas por estos profesores se concentran en el contexto del aula (interacción pedagógica con los alumnos, motivación, planificación, evaluación y escasez de recursos), aspectos que como se está viendo coinciden en general con nuestro estudio. 
BARROS LORENZO, Mónica; Nicolás Martínez, Pilar; Pazos Anido, Marta - Aproximación a los intereses e inquietudes de los profesores... Para lá da tarefa: implicar os estudantes na aprendizagem de línguas estrangeiras no ensino superior. Porto: FLUP, 2019, pp. 44-67 DOI: https://doi.org/10.21747/9789898969217/paraa3

De hecho, en 1999 Flores afirmaba: «apesar da diversidade de contextos escolares onde os professores principiantes se inserem, algumas áreas problemáticas mantêmse» (1999, p. 197) y tras este estudio parafrasearíamos hoy en día esta cita afirmando que a pesar de la diversidad de contextos escolares y de los años transcurridos, la situación se mantiene.

Finalmente, queríamos solo nombrar (porque no es posible profundizar en ello debido a las limitaciones de extensión de esta publicación) que otros documentos en el área de la formación de profesores, como Las competencias clave del profesorado de lenguas segundas y extranjeras y la Parrilla del perfil del profesor de idiomas (EPG), proyectos que en el área del español ha llevado a cabo el Instituto Cervantes, colocan como foco del desarrollo de la competencia docente áreas concomitantes a la categoría «Dar clase» del PEFPl; instrumentos que también han sido utilizados, como ya se ha indicado, por los PeP para la elaboración de sus portafolios reflexivos.

Por todos los motivos expuestos, los resultados a los que se ha llegado apuntan a aspectos que como formadores debemos tener en cuenta y trabajar con más profundidad, tanto en las asignaturas de especialización de los másteres objeto de estudio como durante las sesiones de tutoría y retroalimentación asociadas al comentario y heteroevaluación de las clases de los PeP observadas. A pesar de estas consideraciones generales, el análisis de las reflexiones de los PeP sobre los distintos descriptores anteriormente mencionados aún proporcionará nuevas líneas de estudio que serán abordadas en futuros trabajos.

\section{Referencias bibliográficas}

Almeida, J. et al. (2016). Projetos de Investigação-ação. Orientações gerais para a elaboração do Relatório de Estágio em ensino de Português e da Língua Estrangeira. Porto: Faculdade de Letras da Universidade do Porto. Recuperado de https://bit.ly/2TgpcGi

Cresswell, J. W. (2014). Research Design: Qualitative, Quantitative, \& Mixed Methods Approaches. United Kingdom: Sage Publications.

Decreto-Lei n. 43/2007, de 22 de Fevereiro. Diário da República n. ํ 38/2007, I Série. Ministério da Educação. Lisboa.

Del Rincón, D., Argal, J., Latorre, A. \& Sans, A. (1995). Técnicas de investigación en Ciencias Sociales. Madrid: Editorial DYKINSON.

Despacho n. 10608/2015. Diário da República n.ำ186/2015, I/ Série, de 23 de setembro de 2015. Universidade do Porto - Reitoria. 
BARROS LORENZO, Mónica; Nicolás Martínez, Pilar; Pazos Anido, Marta - Aproximación a los intereses e inquietudes de los profesores... Para lá da tarefa: implicar os estudantes na aprendizagem de línguas estrangeiras no ensino superior. Porto: FLUP, 2019, pp. 44-67 DOI: https://doi.org/10.21747/9789898969217/paraa3

Despacho n. 10609/2015. Diário da República n. 186/2015, II Série, de 23 de setembro de 2015. Universidade do Porto - Reitoria.

Eirín, R., García Ruso, H. Mª \& Montero, L. (2009). Profesores principiantes e iniciación profesional. Estudio Exploratorio. Profesorado. Revista de currículum y formación del profesorado, 13(1), 101-115. Recuperado de https://bit.ly/2Ewrw3F

Faculdade de Letras da Universidade do Porto (2013). Regulamento Geral do Ciclo de Estudos conducente ao Grau de Mestre em Ensino do Português no 3ํ Ciclo do Ensino Básico e no Ensino Secundário e de Língua Estrangeira nos Ensinos Básico e Secundário. Recuperado de https://bit.ly/2E9yXwg

Faculdade de Letras da Universidade do Porto (s.f.). Mestrado em Ensino do Inglês e de Língua Estrangeira no 3ํㅜㄷㅣo do Ensino Básico e no Ensino Secundário, nas áreas de especialização de Alemão ou Espanhol ou FrancêsCiclo de estudos conducente ao grau de Mestre em Ensino de Inglês e de Alemão / Francês / Espanhol no $3^{\circ}$ ciclo do Ensino Básico e no Ensino Secundário. Recuperado de https://bit.ly/2lzMQta

Faculdade de Letras da Universidade do Porto (s.f.). Mestrado em Ensino de Português e de Língua Estrangeira no $3^{\circ}$ Ciclo do Ensino Básico e no Ensino Secundário nas áreas de especialização de Alemão ou Espanhol ou Francês ou InglêsCiclo de estudos conducente ao grau de Mestre em Ensino do Português no 3ํㅗㄷo do Ensino Básico e no Ensino Secundário e de Língua Estrangeira nos Ensinos Básico e Secundários. Recuperado de https://bit.ly/2SYXqOY

Flores, M. A. (1999). (Des)ilusões e paradoxos: A entrada na carreira na perspectiva dos professores neófitos. Revista Portuguesa de Educação, 12(1), 171-204. Recuperado de https://bit.ly/2H1mYDW

Fuller, F. F. (1969). Concerns of Teachers: A Developmental Conceptualization. American Educational Research Journal, 6(2), 207-226.

Fuller, F. \& Brown, O. H. (1975). Becoming a teacher. In K. Ryan (Ed.), Teacher Education (pp. 25-52). Chicago: University of Chicago Press.

Higueras García, M. (2012). Líneas metodológicas para la formación de profesores de lenguas extranjeras. Revista Internacional de Lenguas Extranjeras (RILE), 1, 101128. Recuperado de https://bit.ly/2EwXmxr

Instituto Cervantes (2012). Las Competencias clave del profesorado de lenguas segundas y extranjeras. Madrid: Instituto Cervantes. Recuperado de https://bit.ly/2XR9ZLa

Marcelo, C., Mayor, C. \& Sánchez, M. (1995). Un instrumento para evaluar cambios en las etapas de preocupaciones de profesores: El inventario de preocupaciones de 
BARROS LORENZO, Mónica; Nicolás Martínez, Pilar; Pazos Anido, Marta - Aproximación a los intereses e inquietudes de los profesores... Para lá da tarefa: implicar os estudantes na aprendizagem de línguas estrangeiras no ensino superior. Porto: FLUP, 2019, pp. 44-67 DOI: https://doi.org/10.21747/9789898969217/paraa3

profesores. Enseñanza \& Teaching: Revista interuniversitaria de didáctica, 13, 153-178. Recuperado de https://bit.ly/2MrYkNr

Mateo, J. \& Martínez, F. (2008). Medición y evaluación educativa. Madrid: Editorial La Muralla, S.A.

Newby, D. et al. (2007). Portfolio Europeo para futuros profesores de idiomas. Una herramienta de reflexión para la formación de profesores (PEFPI). Graz: Consejo de Europa, Centro Europeo de Lenguas Modernas. Recuperado de https://bit.ly/2LUUATL

Pastor Cesteros, S. (2012). Portafolio docente y evaluación del profesorado de ELE. In E. Bartol y L. Chamanadjian (Eds.), Actas del Coloquio Internacional sobre la Enseñanza de Español como Lengua Extranjera en Quebec IV, 5-27. Montreal: Universidad de Montreal. Recuperado de https://bit.ly/2NtFgPn

Reyes, L. A. (2011). Profesores, principiantes e inserción profesional a la docencia. Preocupaciones, problemas y desafíos. (Tesis Doctoral Inédita). Universidad de Sevilla, Sevilla. Recuperado de https://bit.ly/2SokbqD

Rodríguez, G., Gil, J. \& García, E. (1996). Metodología de la investigación cualitativa. Málaga: Ediciones Aljibe, S.L.

Ruiz Olabuénaga, J. I. (2007). Metodología de la investigación cualitativa. Bilbao: Universidad de Deusto.

Sabariego, M. \& Bisquerra, R. (2004). El proceso de investigación (parte 1). In R. Bisquerra Alzina (Coord.), Metodología de la investigación educativa (pp. 89-125). Madrid: Editorial La Muralla.

Sabariego, M. (2004). El proceso de investigación (parte 2). In R. Bisquerra Alzina (Coord.), Metodología de la investigación educativa (pp. 128-163). Madrid: Editorial La Muralla.

Sabariego, M., Dorio, I. \& Massot, I. (2004). Métodos de investigación cualitativa. In R. Bisquerra Alzina (Coord.), Metodología de la investigación educativa (pp. 293-328). Madrid: Editorial La Muralla.

Veenman, S. (1984). Perceived Problems of Beginning Teachers. Review of Educational Research, 54, 143-178.

Verdía, E. (2016). ¿Por qué y para qué describir las competencias de los profesores de español? In Olga Cruz Moya (Ed.), La formación y competencias del profesorado de ELE (pp. 37-53). Granada: Asociación para la Enseñanza del Español como Lengua Extranjera. Recuperado de https://bit.ly/2VgobLx

VV. AA. (2013). Parrilla interactiva del perfil del profesor de idiomas (EPG-European Profiling Grid). Recuperado de https://bit.ly/2CBmRvc 